\title{
LncRNA BANCR participates in polycystic ovary syndrome by promoting cell apoptosis
}

\author{
RUIRUI YANG ${ }^{1}$, JIANMEI CHEN ${ }^{1}$, LI WANG $^{1}$ and AIJING DENG ${ }^{2}$ \\ ${ }^{1}$ Department of Obstetrics and Gynecology, Binzhou People's Hospital of Shandong, Binzhou, Shandong 256600; \\ ${ }^{2}$ Department of Gynaecology, The Second Affiliated Hospital of Guangzhou University of Chinese Medicine, \\ Guangdong Provincial Hospital of Chinese Medicine, Guangzhou, Guangdong 510006, P.R. China
}

Received May 1, 2018; Accepted November 21, 2018

DOI: $10.3892 / \mathrm{mmr} .2018 .9793$

\begin{abstract}
The long non-coding RNA (lncRNA) BANCR is a well-studied IncRNA that serves pivotal roles in various malignancies; however, to the best of our knowledge, its involvement in polycystic ovary syndrome (PCOS) remains unknown. In the present study, the expression levels of IncRNA BANCR were detected in granulosa cells (GCs) from patients with PCOS and non-PCOS patients undergoing in vitro fertilization by reverse transcription-quantitative polymerase chain reaction. Subsequently, GCs and the KGN human granulosa-like tumor cell line were treated with insulin, and BANCR expression was detected. KGN cells were also transfected with a BANCR expression vector, after which, cell proliferation, apoptosis and the expression levels of pro-apoptotic B-cell lymphoma 2-associated $\mathrm{X}$ protein (Bax) and p53 were detected by Cell Counting kit- 8 assay, MTT assay and western blotting, respectively. The results revealed that the expression levels of lncRNA BANCR in GCs were significantly higher in patients with PCOS compared with in non-PCOS patients. In addition, insulin treatment significantly upregulated the expression of BANCR in GCs and KGN cells. Transfection with the BANCR expression vector significantly inhibited proliferation and promoted apoptosis of KGN cells, and significantly promoted the expression levels of pro-apoptotic Bax and p53. Therefore, it may be concluded that IncRNA BANCR participates in PCOS by promoting cell apoptosis through the upregulation of Bax and p53.
\end{abstract}

Correspondence to: Dr Aijing Deng, Department of Gynaecology, The Second Affiliated Hospital of Guangzhou University of Chinese Medicine, Guangdong Provincial Hospital of Chinese Medicine, 55 Neihuan West Road, Higher Education Mega Center, Panyu, Guangzhou, Guangdong 510006, P.R. China

E-mail: ocjmba5@163.com

Key words: polycystic ovary syndrome, long non-coding RNA BANCR, B-cell lymphoma 2-associated $\mathrm{X}$ protein, cell proliferation, cell apoptosis

\section{Introduction}

Polycystic ovary syndrome (PCOS) is an endocrine disorder characterized by ovulatory dysfunction, hyperandrogenism and polycystic ovarian morphology; this disorder occurs in $5-20 \%$ of women of reproductive age worldwide (1). PCOS is one of the leading causes of anovulatory infertility (2) and is an important risk factor for type 2 diabetes mellitus (3). However, at present, the pathogenesis of PCOS remains unclear. It is known that abnormal gonadotropin levels may cause anovulation and hyperandrogenism (4). Alterations in ovarian folliculogenesis, which are common in PCOS, are induced by the imbalance between testosterone and follicle-stimulating hormone, caused by excessive production of luteinizing hormone (5). In spite of efforts made regarding the prevention and treatment of PCOS, long-term outcomes remain generally unsatisfactory. Therefore, novel targets are required to improve the clinical treatment outcomes of PCOS.

PCOS is caused by elevated androgens and is characterized by altered cell proliferation, differentiation, steroidogenesis, follicle maturation and apoptosis (6). Numerous intra- and extra-ovarian factors, including tumor necrosis factor $\alpha$, vascular endothelial growth factor, epidermal growth factors and interleukins have been reported to be involved in the pathogenesis of PCOS (6). Alongside mRNAs that encode protein products, the human genome transcribes a larger set of non-coding RNAs (ncRNAs) (7). Long ncRNAs (IncRNAs) are a subgroup of ncRNAs composed of $>200$ nucleotides, which serve critical roles in the pathogenesis of various human diseases (8). It has been reported that the development of PCOS is accompanied by alterations in the expression levels of certain lncRNAs (9), thus indicating the involvement of IncRNAs in this disease. LncRNA BANCR is a recently identified IncRNA that has pivotal roles in various malignancies, including endometrial cancer (10) and melanoma (11), mainly by promoting the proliferation of cancer cells through interactions with numerous pathways. Our preliminary experiments detected altered expression of BANCR in PCOS tissues compared with in healthy controls (data not shown). The present study aimed to examine the potential involvement of IncRNA BANCR in PCOS. It was demonstrated that lncRNA BANCR may participate in PCOS 
by promoting cell apoptosis through the upregulation of $\mathrm{B}$-cell lymphoma (Bcl)-2-associated X protein (Bax).

\section{Patients and methods}

Subjects and granulosa cells (GCs). The present study recruited 44 patients with PCOS; these patients were diagnosed and treated for the first time at The Second Affiliated Hospital of Guangzhou University of Chinese Medicine, Guangdong Provincial Hospital of Chinese Medicine (Guangdong, China) between January 2016 and January 2017. The patients were aged between 22 and 39 years old, with a mean age of $31.2 \pm 3.4$ years. GCs were collected from each patient. Briefly, GCs were separated from whole blood samples via centrifugation at $1,875 \mathrm{x} g$ and room temperature for $10 \mathrm{~min}$ in tubes containing $80 \%$ Percoll; GCs formed a layer on the Percoll solution. GCs were also collected from non-PCOS patients ( $\mathrm{n}=34$; age range, 21-39; mean, 30.9 \pm 3.1 ) undergoing in vitro fertilization, which served as a control. GCs were cultured in Dulbecco's modified Eagle's medium (DMEM)/F12 (1:1; Gibco; Thermo Fisher Scientific, Inc., Waltham, MA, USA) containing $10 \%$ fetal bovine serum (FBS, Sigma-Aldrich; Merck KGaA, Darmstadt, Germany) for 3 days prior to experimentation. There was no significant difference in age between the patient and control groups. The present study was approved by the ethics committee of The Second Affiliated Hospital of Guangzhou University of Chinese Medicine, Guangdong Provincial Hospital of Chinese Medicine. All patients provided written informed consent.

Human granulosa-like tumor cell line (KGN) and transfection. The KGN human granulosa-like tumor cell line was recently established in our laboratory using tissue samples from a patient with invasive ovarian granulosa cell carcinoma, according to methods described by Nishi et al (12). KGN cells were cultured in DMEM/F12 (1:1) containing 10\% FBS. BANCR cDNA was amplified from cDNA derived from the total RNA of GCs using primers carrying the EcoRI restriction enzyme sites at the flanking ends. The EcoRI-EcoRI fragment containing BANCR cDNA was inserted into the GV299 lentiviral vector (Shanghai GeneChem Co., Ltd., Shanghai, China) to establish a BANCR expression vector. KGN cells were cultured overnight to reach $80-90 \%$ confluence and $10 \mathrm{nM}$ vectors were transfected into cells. Empty vectors without BANCR cDNA were also transfected into KGN cells to serve as negative controls.

Total RNA extraction and reverse transcription-quantitative polymerase chain reaction $(R T-q P C R)$. TRIzol ${ }^{\circledR}$ Reagent (Invitrogen; Thermo Fisher Scientific, Inc.) was used to extract total RNA from GCs and KGN cells. To detect the effects of insulin on BANCR, GCs were first treated with insulin (5, 10 and $50 \mathrm{ng} / \mathrm{ml}$; Sigma-Aldrich; Merck KGaA) for $24 \mathrm{~h}$ at $37^{\circ} \mathrm{C}$ in a $5 \% \mathrm{CO}_{2}$ prior to RNA extraction. Total RNA was quantified using a NanoDrop ${ }^{\mathrm{TM}} 2000$ Spectrophotometer (NanoDrop; Thermo Fisher Scientific, Inc., Wilmington, DE, USA), and RNA samples with an A260/A280 ratio of 1.8-2.0 were used to synthesize cDNA through RT using SuperScript

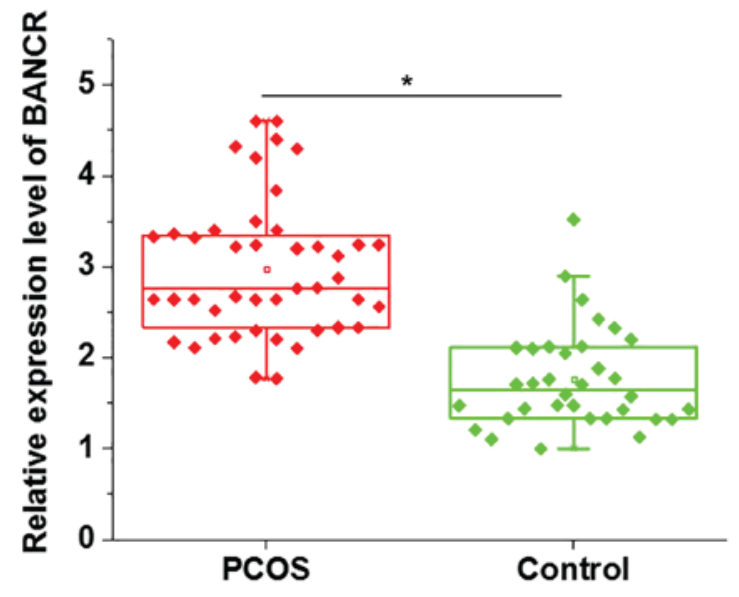

Figure 1. Comparison of BANCR expression in GCs between patients with PCOS and controls. The results of reverse transcription-quantitative polymerase chain reaction demonstrated that the expression levels of BANCR were significantly higher in GCs in patients with PCOS than in controls. ${ }^{*} \mathrm{P}<0.05$. GCs, granulosa cells; PCOS, polycystic ovary syndrome.

III Reverse Transcriptase kit (Thermo Fisher Scientific, lnc.) according to manufacturer's instructions. PCR reaction systems were prepared using SYBR ${ }^{\circledR}$ Green Quantitative RT-qPCR kit (Sigma-Aldrich; Merck KGaA). PCR reactions were performed on an ABI 7500 system (Applied Biosystems; Thermo Fisher Scientific, Inc., Waltham, MA, USA). Primers used in PCR reactions were as follows: BANCR forward, 5'-ACAGGACTCCATGGCAAACG-3' and reverse 5'-ATGAAGAAAGCCTGGTGCAGT-3'; and $\beta$-actin forward, 5'-GACCTCTATGCCAACACAGT-3' and reverse, 5'-AGTACTTGCGCTCAGGAGGA-3'. qPCR was carried out using the following thermocycling conditions: $95^{\circ} \mathrm{C}$ for $50 \mathrm{sec}$, followed by 40 cycles at $95^{\circ} \mathrm{C}$ for $12 \mathrm{sec}$ and $60^{\circ} \mathrm{C}$ for $45 \mathrm{sec}$. All data were processed using the $2^{-\Delta \Delta \mathrm{Cq}}$ method (13). Relative expression levels of BANCR were normalized to the endogenous control $\beta$-actin.

Cell proliferation assay. Cell Counting kit-8 (Dojindo Molecular Technologies, Inc., Kumamoto, Japan) was performed to measure the proliferation of KGN cells with or without BANCR overexpression, according to the manufacturer's protocol. Briefly, 3,000 cells $/ 100 \mu 1$ were added to each well of a 96 -well plate. Cells were cultured at $37^{\circ} \mathrm{C}$ in a $5 \% \mathrm{CO}_{2}$ incubator, and $10 \mu \mathrm{l} \mathrm{CCK-8}$ was added to each well after 24 , 48, 72 and $96 \mathrm{~h}$ later. Cells were incubated with CCK-8 for $4 \mathrm{~h}$ at $37^{\circ} \mathrm{C}$. Finally, an Epoch Microplate Spectrophotometer (BioTek Instruments, Inc., Winooski, VT, USA) was used to measure optical density values at $450 \mathrm{~nm}$. Cell proliferation was normalized to control cells.

Cell apoptosis assay. Cells were suspended in serum-free medium $\left(6 \times 10^{4}\right.$ cells $\left./ \mathrm{ml}\right)$ and were transferred to a 6-well plate (10 $\mathrm{ml}$ cell suspension/well). After cell culture for $48 \mathrm{~h}$, cells were subjected to $0.25 \%$ trypsin digestion. Subsequently, cells were stained with Annexin V-fluorescein isothiocyanate (FITC; 1:50; Dojindo Molecular Technologies, Inc.) and propidium iodide (PI, 1:50). Flow cytometry was performed to detect apoptotic cells. Data were analyzed using BD FACSuite ${ }^{\mathrm{TM}}$ software version 1.0 (BD Biosciences, San Jose, CA, USA). 
A

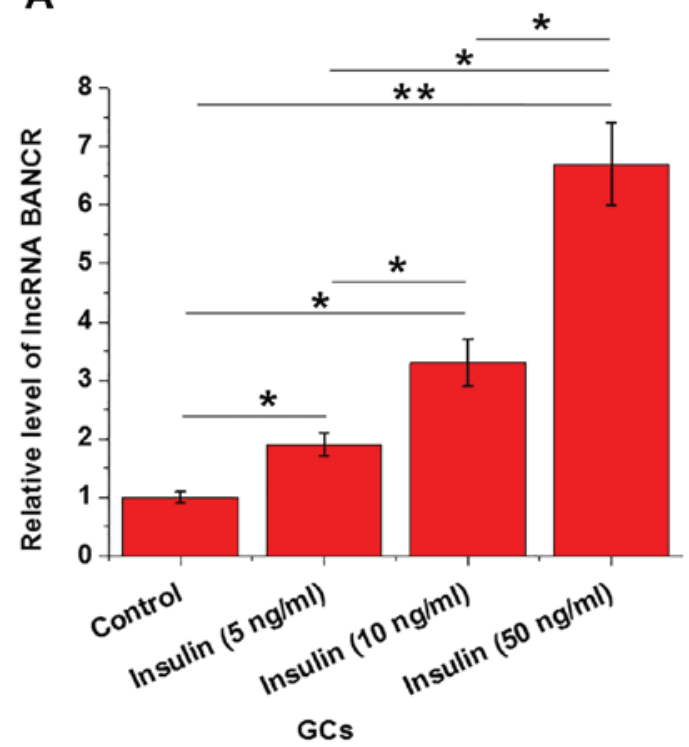

B

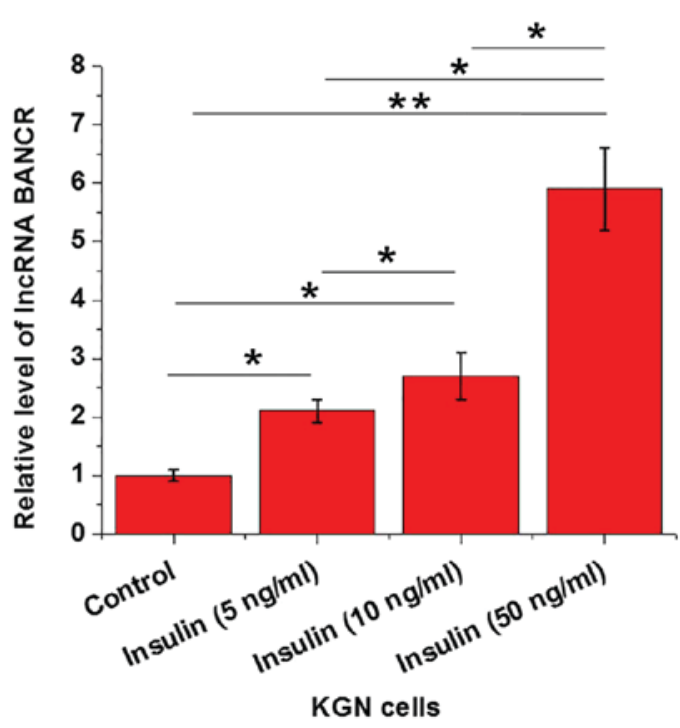

Figure 2. Effects of insulin treatment on BANCR expression in GCs and KGN cells. The results of reverse transcription-quantitative polymerase chain reaction demonstrated that insulin treatment significantly upregulated the expression levels of BANCR in (A) GCs and (B) KGN cells in a dose-dependent manner. ${ }^{*} \mathrm{P}<0.05,{ }^{* *} \mathrm{P}<0.01$. GCs, granulosa cells; lncRNA, long non-coding RNA.

Cell apoptotic rates were calculated based on the sum of $\%$ Annexin V-FITC ${ }^{+} / \mathrm{PI}$ - and $\%$ Annexin V-FITC ${ }^{+} / \mathrm{PI}^{+}$cells.

Western blotting. Radioimmunoprecipitation assay buffer (Thermo Fisher Scientific, Inc., Waltham, MA, USA) was used to extract total proteins from KGN cells. Bicinchoninic acid assay was used to quantify protein samples. Subsequently, proteins (30 $\mu \mathrm{g} /$ lane) were separated by $10 \%$ SDS-PAGE and were transferred to polyvinylidene fluoride membranes, which were blocked with $5 \%$ skimmed milk at room temperature for $1 \mathrm{~h}$. Tris-buffered saline-3\% Tween-20 (TBST) was used to wash the membranes three times (5 min/wash), after which, membranes were incubated with the following primary antibodies: Rabbit anti-Bax antibody (1:2,000, cat. no. ab32503), anti-p53 (1:2,000, cat. no. ab31333) and anti-GAPDH (1:1,000, cat. no. ab9485; all Abcam, Cambridge, UK) overnight at $4^{\circ} \mathrm{C}$. Subsequently, TBST was used to wash the membranes three times ( $5 \mathrm{~min} /$ wash), followed by incubation with an anti-rabbit immunoglobulin G-horseradish peroxidase secondary antibody $(1: 1,000$; cat. no. MBS435036; MyBioSource, Inc., San Diego, CA, USA) at room temperature for $1 \mathrm{~h}$. TBST was used to wash the membranes a further three times ( $5 \mathrm{~min} /$ wash). Enhanced chemiluminescence (Sigma-Aldrich; Merck KGaA, Darmstadt, Germany) was then used to develop the signal. Relative expression levels were normalized to the endogenous control GAPDH using ImageJ version 1.47 (National Institutes of Health, Bethesda, MD, USA).

Statistical analysis. SPSS 19.0 (IBM Corp., Armonk, NY, USA) was used to analyze results. Experiments were performed in triplicate. Data are expressed as the means \pm standard deviation; comparisons among multiple groups were determined using one-way analysis of variance followed by the least significant difference post hoc test, whereas differences between two groups were analyzed by Student's t-test. $\mathrm{P}<0.05$ was considered to indicate a statistically significant difference.

\section{Results}

Comparison of BANCR expression in GCs between patients with PCOS and controls. The mRNA expression levels of BANCR in GCs derived from patients with PCOS and healthy controls were detected by RT-qPCR. As shown in Fig. 1, the expression levels of BANCR were significantly higher in GCs from patients with PCOS than in controls. These data suggested that BANCR may be involved in the pathogenesis of PCOS.

Effects of insulin treatment on BANCR expression in GCs and $K G N$ cells. Insulin treatment was performed by adding various concentrations of insulin (5, 10 and $50 \mathrm{ng} / \mathrm{ml}$; Sigma-Aldrich; Merck KGaA) into the culture media. As presented in Fig. 2, insulin treatment significantly upregulated the expression levels of BANCR in GCs (Fig. 2A) and KGN cells (Fig. 2B) in a dose-dependent manner.

Effects of BANCR overexpression on proliferation and apoptosis of KGN cells. CCK-8 assay and MTT assay were performed to investigate the effects of BANCR overexpression on the proliferation and apoptosis of KGN cells. BANCR vector significantly increased BANCR expression, compared with the controls (Fig. 3A). As shown in Fig. 3B, compared with control cells and negative control cells, BANCR overexpression significantly inhibited the proliferation of $\mathrm{KGN}$ cells $(\mathrm{P}<0.05)$. In addition, BANCR overexpression significantly promoted apoptosis of $\mathrm{KGN}$ cells (Fig. 3C; $\mathrm{P}<0.05$ ).

Effects of BANCR overexpression on Bax and p53 expression in KGN cells. Bax and p53 expression in KGN cells with or 
A

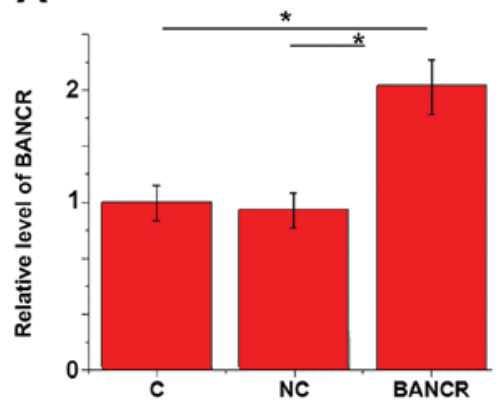

B

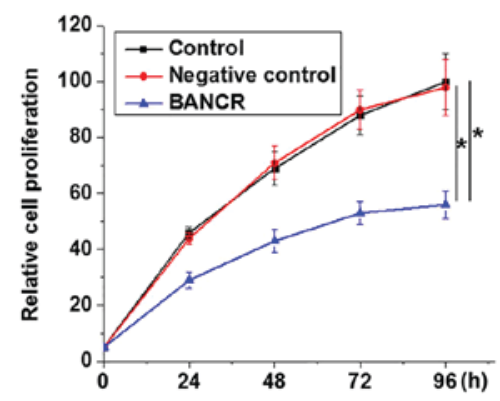

C

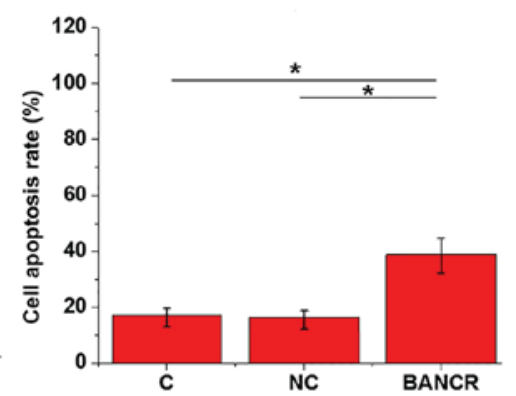

Figure 3. Effects of BANCR overexpression on the proliferation and apoptosis of KGN cells. (A) BANCR overexpression was confirmed in KGN cells by reverse transcription-quantitative polymerase chain reaction. The results of Cell Counting kit- 8 and cell apoptosis assays indicated that BANCR overexpression significantly (B) inhibited proliferation and (C) promoted apoptosis of KGN cells. "P<0.05. C, control (untreated cells); NC, negative control.

A

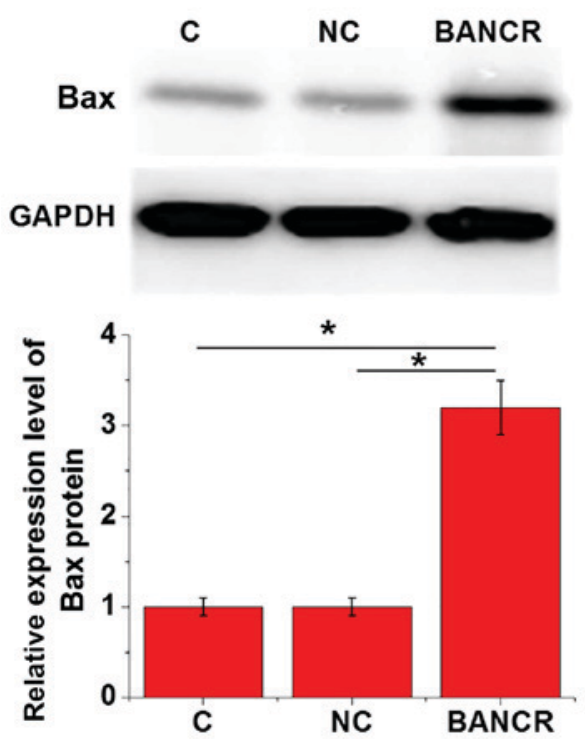

B

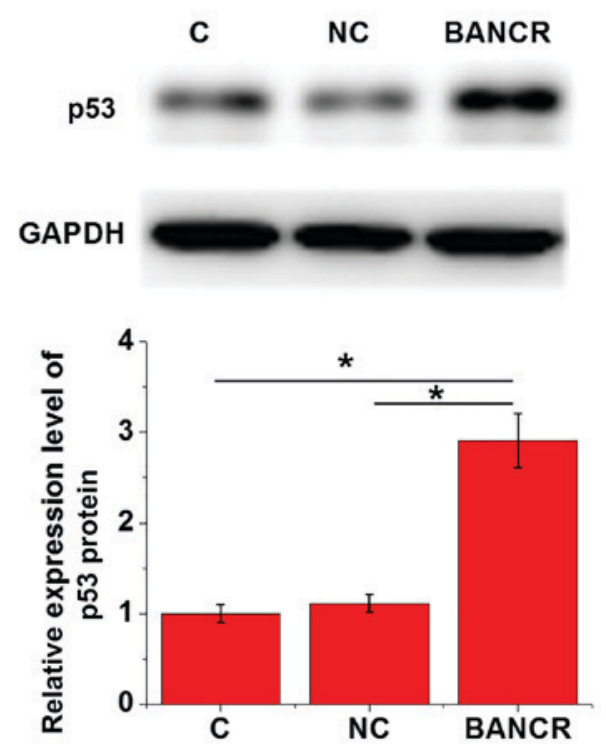

Figure 4. Effects of BANCR overexpression on Bax and p53 expression in KGN cells. Western blotting revealed that, compared with in control and NC cells, BANCR overexpression significantly upregulated (A) Bax and (B) p53 expression in KGN cells. "P<0.05. Bax, B-cell lymphoma 2-associated X protein; $\mathrm{C}$, control; NC, negative control.

without BANCR overexpression was detected by western blotting. As shown in Fig. 4, compared with in the control and negative control cells, BANCR overexpression significantly upregulated Bax (Fig. 4A) and p53 (Fig. 4B) expression in KGN cells $(\mathrm{P}<0.05)$.

\section{Discussion}

The present study reported a potential novel function of lncRNA BANCR, which has well-characterized functions in human malignancies. The results demonstrated that lncRNA BANCR may be upregulated by pathological insulin signaling, and it may promote the development of PCOS by promoting apoptosis and inhibiting proliferation of GCs by activating the Bax pro-apoptotic pathway.

It has previously been reported that the development of PCOS is associated with the abnormal expression of numerous genetic factors (6). However, to the best of our knowledge, the involvement of lncRNAs in PCOS has not yet been well studied. In a recent study, Liu et al reported that the expression levels of
lncRNA steroid receptor RNA activator RNA activator (SRA) in peripheral blood leukocytes is closely correlated with the development of PCOS (14), whereas the function of lncRNA SRA and the mechanism of its action in PCOS are unknown. It has been well established that GCs have a key role in folliculogenesis, and are essential for the maintenance of a suitable microenvironment for the initiation of follicle growth, oocyte maturation and atresia (15). In the present study, the expression levels of lncRNA BANCR were significantly upregulated in patients with PCOS compared with in controls, thus indicating that upregulation of BANCR may be involved in the pathogenesis of PCOS.

The majority of patients with PCOS will develop insulin resistance, causing cardiovascular disease, hyperinsulinemia and an increased risk for type 2 diabetes (16). Insulin signaling is a key player in the regulation of hormone production in GCs, as well as regulation of ovarian function and GC proliferation $(17,18)$. In the present study, insulin treatment significantly promoted the expression of IncRNA BANCR in GCs and KGN cells in a dose-dependent manner. These data suggested 
that lncRNA BANCR may be involved in pathological insulin signaling to promote the progression of PCOS. Therefore, BANCR may serve as a target for the prevention of type 2 diabetes in patients with PCOS.

Unbalanced proliferation and apoptosis of GCs may lead to abnormal folliculogenesis $(19,20)$. The KGN cell line has been widely used to investigate cell growth and apoptosis of human GCs (12). In the present study, BANCR overexpression significantly promoted apoptosis and inhibited proliferation of KGN cells, thus indicating that BANCR may participate in the pathogenesis of PCOS by stimulating apoptosis and inhibiting proliferation of GCs. Bax-mediated cell apoptosis serves a pivotal role in the development of various types of human disease $(21,22)$. A recent study also reported that expression levels of Bax are significantly upregulated in patients with PCOS compared with in healthy controls (23). In this study, BANCR overexpression significantly upregulated the protein expression levels of Bax in KGN cells, indicating that the enhancing effects of BANCR overexpression on apoptosis of KGN cells may be achieved by upregulating Bax expression.

Notably, other apoptosis-associated proteins, including insulin-like growth factors (IGFs), IGF-binding proteins, Bcl-2 and Bcl-extra large were also detected in this study (data not shown); however, no significant alterations in the expression levels of these proteins were detected following BANCR overexpression. Our future studies aim to focus on other pathways involved in BANCR-mediated regulation of KGN cell apoptosis.

In conclusion, IncRNA BANCR expression was upregulated in GCs from patients with PCOS. In addition, insulin treatment significantly upregulated the expression of BANCR in GCs and KGN cells. BANCR overexpression significantly inhibited proliferation and promoted apoptosis of KGN cells, and upregulated the expression levels of pro-apoptotic Bax and p53 in KGN cells. These findings indicated that lncRNA BANCR may participate in PCOS by promoting cell apoptosis through the upregulation of Bax.

\section{Acknowledgements}

Not applicable.

\section{Funding}

The authors are grateful for the financial support they received in 2014 from The Scientific Research Project of Guangdong Provincial Administration of Traditional Chinese Medicine, for the project 'The research of acupuncture point buried line combined with daily lifestyle intervention to treat polycystic ovary syndrome' (project no. 20141124, project support).

\section{Availability of data and materials}

All data generated or analyzed during this study are included in this published article.

\section{Author's contributions}

RY, JC, LW and AD were responsible for the conception and design of the study. RY and JC performed the experiments. RY, JC and LW analyzed and interpreted the data. RY drafted the article. JC, LW and AD were responsible for the revision of the manuscript.

\section{Ethics approval and consent to participate}

The present study was approved by the Ethics Review Committee of The Second Affiliated Hospital of Guangzhou University of Chinese Medicine, Guangdong Provincial Hospital of Chinese Medicine. All patients provided written informed consent.

\section{Patient consent for publication}

Not applicable.

\section{Competing interests}

The authors declare that they have no competing interests.

\section{References}

1. Azziz R, Carmina E, Chen ZJ, Dunaif A, Laven JS, Legro RS, Lizneva D, Natterson-Horowtiz B, Teede HJ and Yildiz BO: Polycystic ovary syndrome. Nat Rev Dis Primers 2: 16057, 2016.

2. Franks S: Polycystic ovary syndrome. Medicine 45: 527-531, 2017.

3. Ollila MM, West S, Keinänen-Kiukaanniemi S, Jokelainen J, Auvinen J, Puukka K, Ruokonen A, Järvelin MR, Tapanainen JS, Franks S, et al: Overweight and obese but not normal weight women with PCOS are at increased risk of Type 2 diabetes mellitus-a prospective, population-based cohort study. Hum Reprod 32: 423-431, 2017.

4. Diamanti-Kandarakis E and Dunaif A: Insulin resistance and the polycystic ovary syndrome revisited: An update on mechanisms and implications. Endocr Rev 33: 981-1030, 2012.

5. Franks S, Stark J and Hardy K: Follicle dynamics and anovulation in polycystic ovary syndrome. Hum Reprod Update 14: 367-378, 2008

6. De Leo V, Musacchio MC, Cappelli V, Massaro MG, Morgante G and Petraglia F: Genetic, hormonal and metabolic aspects of PCOS: An update. Reprod Biol Endocrinol 14: 38, 2016.

7. Mattick JS and Makunin IV: Non-coding RNA. Hum Mol Genet 15 Spec No 1: R17-R29, 2006.

8. Wapinski O and Chang HY: Long noncoding RNAs and human disease. Trends Cell Biol 21: 354-361, 2011.

9. Huang X, Hao C, Bao H, Wang M and Dai H: Aberrant expression of long noncoding RNAs in cumulus cells isolated from PCOS patients. J Assist Reprod Genet 33: 111-121, 2016.

10. Wang D, Wang D, Wang N, Long Z and Ren X: Long non-coding RNA BANCR promotes endometrial cancer cell proliferation and invasion by regulating MMP2 and MMP1 via ERK/MAPK signaling pathway. Cell Physiol Biochem 40: 644-656, 2016.

11. Li R, Zhang L, Jia L, Duan Y, Li Y, Bao L and Sha N: Long non-coding RNA BANCR promotes proliferation in malignant melanoma by regulating MAPK pathway activation. PLoS One 9: e100893, 2014.

12. Nishi Y, Yanase T, Mu YM, Oba K, Ichino I, Saito M, Nomura M, Mukasa C, Okabe T, Goto K, et al: Establishment and characterization of a steroidogenic human granulosa-like tumor cell line, KGN, that expresses functional follicle-stimulating hormone receptor. Endocrinology 142: 437-445, 2001.

13. Livak KJ and Schmittgen TD: Analysis of relative gene expression data using real-time quantitative PCR and the 2(-Delta Delta C(T)) method. Methods 25: 402-408, 2001.

14. Liu Z, Hao C, Huang X, Zhang N, Bao H and Qu Q: Peripheral blood leukocyte expression level of lncRNA steroid receptor RNA activator SRA. and its association with polycystic ovary syndrome: A case control study. Gynecol Endocrinol 31: 363-368, 2015.

15. Eppig JJ: Oocyte control of ovarian follicular development and function in mammals. Reproduction 122: 829-838, 2001. 
16. Legro RS, Finegood D and Dunaif A: A fasting glucose to insulin ratio is a useful measure of insulin sensitivity in women with polycystic ovary syndrome. J Clin Endocrinol Metab 83: 2694-2698, 1998.

17. Wang L, Li H, Yang S, Ma W, Liu M, Guo S, Zhan J, Zhang H, Tsang SY, Zhang Z, et al: Cyanidin-3-o-glucoside directly binds to ER $\alpha 36$ and inhibits EGFR-positive triple-negative breast cancer. Oncotarget 7: 68864-68882, 2016.

18. Ni XR, Sun ZJ, Hu GH and Wang RH: High concentration of insulin promotes apoptosis of primary cultured rat ovarian granulosa cells via its increase in extracellular HMGB1. Reprod Sci 22: 271-277, 2015.

19. Das M, Djahanbakhch O, Hacihanefioglu B, Saridogan E, Ikram M, Ghali L, Raveendran M and Storey A: Granulosa cell survival and proliferation are altered in polycystic ovary syndrome. J Clin Endocrinol Metab 93: 881-887, 2008.

20. Onalan G, Selam B, Baran Y, Cincik M, Onalan R, Gündüz U, Ural AU and Pabuccu R: Serum and follicular fluid levels of soluble Fas, soluble Fas ligand and apoptosis of luteinized granulosa cells in PCOS patients undergoing IVF. Hum Reprod 20: 2391-2395, 2005.
21. Gillissen B, Richter A, Richter A,Preissner R, Schulze-Osthoff K Essmann F and Daniel PT: Bax/Bak-independent mitochondrial depolarization and reactive oxygen species induction by sorafenib overcome resistance to apoptosis in renal cell carcinoma. J Biol Chem 292: 6478-6492, 2017.

22. Mei S, Li L, Wei Q, Hao J, Su Y, Mei C and Dong Z: Double knockout of Bax and Bak from kidney proximal tubules reduces unilateral urethral obstruction associated apoptosis and renal interstitial fibrosis. Sci Rep 7: 44892, 2017.

23. Wu XQ, Wang YQ, Xu SM, Liu JF, Bi XY, Wang ZQ and Zhang JP: The WNT/ $\beta$-catenin signaling pathway may be involved in granulosa cell apoptosis from patients with PCOS in North China. J Gynecol Obstet Hum Reprod 46: 93-99, 2017. Attribution 4.0 International (CC BY-NC 4.0) License 